\begin{tabular}{|c|c|}
\hline $\begin{array}{l}\text { REVISTA } \\
\text { EDUEACIÓN }\end{array}$ & $\begin{array}{l}\text { Revista Educación } \\
\text { ISSN: 0379-7082 } \\
\text { ISSN: } 2215-2644 \\
\text { revedu@gmail.com } \\
\text { Universidad de Costa Rica } \\
\text { Costa Rica }\end{array}$ \\
\hline
\end{tabular}

\title{
Rendimiento académico universitario y asistencia a clases: Una visión
}

\author{
Gabalán Coello, Jesús; Vásquez Rizo, Fredy-Eduardo \\ Rendimiento académico universitario y asistencia a clases: Una visión \\ Revista Educación, vol. 41, núm. 2, 2017 \\ Universidad de Costa Rica, Costa Rica \\ Disponible en: http://www.redalyc.org/articulo.oa?id=44051357002 \\ DOl: http://dx.doi.org/10.15517/revedu.v41i2.18477
}

Esta obra está bajo una Licencia Creative Commons Atribución-NoComercial-SinDerivar 3.0 Internacional. 


\section{Rendimiento académico universitario y asistencia a clases: Una visión}

\section{University Academic Achievement and Attendance: a Vision}

Jesús Gabalán Coello [1]

Universidad Autónoma de Occidente, Colombia

DOI: http://dx.doi.org/10.15517/revedu.v41i2.18477

jgabalan@uao.edu.co

Fredy-Eduardo Vásquez Rizo [2]

Universidad Autónoma de Occidente, Colombia

fvasquez@uao.edu.co

Recepción: 13 Marzo 2015

Aprobación: 01 Diciembre 2016

\section{Resumen:}

El presente estudio plasma una serie de reflexiones en torno a la creciente necesidad en el contexto educativo de identificar aspectos que interactúan como condicionantes del rendimiento estudiantil. Investigaciones en esta materia han determinado la relevancia de variables tanto endógenas como exógenas. Este segundo grupo de variables por lo general presentan las condiciones ligadas al proceso de enseñanza-aprendizaje propiamente dicho, tales como: relaciones interpersonales estudiante-estudiante, estudiantedocente, metodologías de enseñanza, esquemas de seguimiento y evaluación; etc. Con cada vez mayor frecuencia, surgen algunas posturas en las cuales se intenta atribuir relevancia a las variables de asistencia o ausentismo a clases. Este texto expone una revisión sobre los enfoques en referencia a la relación existente entre asistencia y rendimiento académico y toma en consideración un análisis de caso al interior de una universidad colombiana. Encontró, en algunos casos, niveles de incidencia entre la asistencia a clases y las notas definitivas, condicionados a ciertas asignaturas, en estudiantes de primero a cuarto semestre. Además, el estudio involucra otra serie de variables, necesarias para poder fortalecer dicha postura, tales como: tamaño del curso, tasa de mortalidad histórica de la asignatura y percepciones del alumnado con respecto a la evaluación docente, identificando su ponderación con respecto al rendimiento general.

Palabras ClaVE: Asistencia a clases, desempeño académico, análisis multivariado, métodos cuantitativos, asistencia obligatoria.

\section{Abstract:}

The present study captures a series of reflections on the growing need in the educational context to identify aspects that interact as conditions of student achievement. Research in this area has determined the relevance of both endogenous and exogenous variable. In this second group of variable, conditions attached to the teaching-learning process itself usually appear, such as student to student relationships, student to teacher relationships, teaching methodologies, monitoring and evaluation schemes; etc.; some positions appear with increasing frequency, which one tries to attribute relevance to the variables of attendance or absence in classes. This test presents a review of approaches concerning the relationship between attendance and performance and takes into consideration a case study within a Colombian university, finding, in some cases, there are correlations between school attendance and final grades, conditional to certain subjects, the first through fourth semester. In attrition, the study involves another set of variables which are necessary to strengthen this position, such as class size, historical mortality rate of the subject, and the students' perceptions regarding teacher evaluation, identifying its weighting with respect with general performance.

KEYWORDS: Class attendance, academic achievement, multivariate analysis, quantitative methods, mandatory attendance.

\section{NotAs DE AUTOR}

[1] Ingeniero industrial por la Universidad Autónoma de Occidente (UAO), Colombia, Magíster en Ingeniería con énfasis Industrial por la Universidad del Valle, Colombia, Ph. D en Medición y Evaluación en Educación por la Universidad de Montreal, Canadá. Ha desarrollado su actividad académica y profesional en las áreas de aseguramiento de la Calidad, Planeación, Investigación y Evaluación. Es profesor de investigación de operaciones y estadística, y se desempeña como coordinador de calidad en la Oficina de Planeación y Desarrollo Institucional de la UAO, en cuya universidad es miembro del Grupo de Investigación en Educación. Ha participado como autor y coautor de varias ponencias y artículos científicos de calidad, evaluación y estadística, publicados en revistas nacionales e internacionales. Es par evaluador del Consejo Nacional de Acreditación (CNA).

[2] Comunicador social-periodista por la Universidad Autónoma de Occidente (UAO), Colombia, y Magister en Ciencias de la Información y Administración del Conocimiento por el Instituto Tecnológico y de Estudios Superiores de Monterrey (Itesm), México, Estudiante de Doctorado en Gestión de la Información y la Comunicación en las Organizaciones por la Universidad de Murcia, España. Trabaja como coordinador de sistemas de información de la Oficina de Planeación y Desarrollo Institucional de la UAO, docente del Área de Comunicación; coordinador y miembro activo de los grupos de investigación en Gestión del Conocimiento y Sociedad de la Información y Educación, respectivamente, en la misma institución. Ha elaborado diversos proyectos, tres libros, artículos en Colombia, España, Argentina y México, cuatro capítulos de libro (uno para Pnuma y Colciencias) y varias ponencias. Es par evaluador de Colciencias. 


\section{INTRODUCCIÓN[3]}

En el sistema convencional de enseñanza en pregrado es recurrente la utilización de conferencias o encuentros de clase como herramienta facilitadora del proceso de aprendizaje. En esta dirección, la asistencia a clases aparece como concepto relevante, pues los procesos educativos que involucran al alumnado en su contexto tienden a garantizar un mayor éxito en los desarrollos de formación (Trigwell y Prosser, 2000). Este fenómeno ha generado, de manera consulta o inconsulta, la aparición de políticas en torno a la obligatoriedad o autonomía en la asistencia a clases.

Estas políticas acontecen en algunos contextos de estudios que tengan en cuenta las particularidades del entorno en el cual se educa, características culturales del país o región, de la institución de educación superior y, por supuesto, de una sólida revisión de lo que se ha hecho con anterioridad sobre este tema en otras instituciones y sistemas educativos trasnacionales.

El estudio que aquí se propone toma en consideración una revisión de los principales aportes en el tema hecho por los estudios más representativos. Esta fase aboga por la comprensión del fenómeno desde su dimensión global y sus principales alcances, hallazgos y restricciones.

La segunda fase del trabajo contextualiza la política de asistencia obligatoria y su impacto directo en los eventos totales de asignación y estudiantes que han sufrido el rigor de la medida.

En fases posteriores, se intenta determinar la posible relación existente entre los niveles de asistencia y los desempeños académicos estudiantiles al final de un curso; así como la inclusión de variables de curso que probablemente interactúan con los niveles de asistencia condicionando las notas definitivas obtenidas.

La justificación de la pertinencia científica de esta investigación encuentra su argumento en la necesidad de la comunidad académica por investigar constantemente los aspectos que tienen una influencia directa en el desempeño académico. Las investigaciones anteriores en la materia han encontrado divergencias sobre la obligatoriedad alrededor de la asistencia a clases y, por tanto, es un tema que necesita ser estudiado teniendo en cuenta, además, otras variables que pueden estar asociadas con la relación parcial entre asistencia y rendimiento.

Por tanto, la siguiente pregunta orienta esta investigación: ¿son determinantes los niveles de asistencia en el posterior rendimiento académico del estudiantado?

\section{Marco teórico}

La comunidad académica se encuentra con frecuencia dividida sobre las políticas relacionadas con la asistencia obligatoria. De acuerdo con St. Clair (1999), esta teme que altos índices de inasistencia inclinen la percepción del estudiantado a pensar que los cursos han sido de baja calidad. A su vez, esta percepción de los cursos afecta negativamente las valoraciones alrededor de la calidad de la universidad y amenaza futuros procesos de admisión, financiamiento y, en términos generales, la estabilidad y el futuro institucional, pues como afirma Ramsden (1991), todo esto puede llegar a influir en la percepción del rendimiento estudiantil, del mismo personal docente, de la unidad académica a la cual pertenece y hasta de su propia institución. Por consiguiente, numerosas investigaciones han intentado determinar la relación entre asistencia a clase y rendimiento académico.

Pero la pregunta acerca del absentismo es en nuestros días una preocupación mundial, pues se presume una fuerte correlación positiva entre mejores resultados académicos de estudiantes y asistencia activa en las clases (Expósito y De la Iglesia, 2010). En Francia, por ejemplo, se ha promulgado una ley por parte de los diputados para luchar contra este fenómeno. Hoy por hoy, es objeto de controversia que quienes defienden la prevención se opongan a quienes la reprimen, situación compleja en su análisis debido a que las raíces del problema son, evidentemente, polimórficas. Al respecto, Dayo (2011), determina que cuando la decisión de 
asistir a clases es potestad del estudiantado, este es más propenso a no presentarse; por lo tanto, las medidas de asistencia obligatoria deben centrarse en disminuir dicha percepción de control.

Existen investigaciones que experimentalmente han concluido la nula o poca asociación entre asistencia y rendimiento. Por ejemplo, Hyde y Flournoy (1986), no encontraron la significancia en el coeficiente de Pearson a través de las dos variables analizadas. Afirman, además, que no es un requerimiento la asistencia a clases para tener indicadores de éxito en el curso; por esto las reglamentaciones asociadas a la asistencia obligatoria podrían afectar negativamente el desempeño académico de alguna parte del estudiantado.

Lo anterior es corroborado por San Martín-Gutiérrez, Jiménez-Torres y Jerónimo-Sánchez-Beato (2016), quienes afirman en un estudio reciente, realizado en el Espacio Europeo de Educación Superior, que incluir la asistencia dentro de los parámetros de evaluación carece de sentido, pues consideran que este hecho tiene poca relación con el rendimiento final estudiantil (situación respaldada por el 94,1\% de las universidades privadas europeas analizadas).

Por su parte, Berenson, Carter y Norwood (1992), implementaron una normativa coercitiva para la asistencia, mostrando un mejoramiento en dicha variable, mas no así en el rendimiento académico, como factor predecible a través de la presencia del estudiantado en el aula. Además, señalaron que existen diversos motivos, independientes de la asistencia, que configuran a un estudiantado de bajo rendimiento y, por tanto, no es garantía que solamente reglamentos de control contribuyan al éxito académico para todo el alumnado.

En este mismo sentido, Levine y Renelt (1992), identificó que existía una correlación negativa moderada entre el número de asistencias y el rendimiento, sugiriendo que coexistían variables intermediarias, tales como el grado en que los exámenes reflejaban el material presentado en clase o factores motivacionales.

Por otro lado, se encuentra una serie de contraargumentaciones en este sentido; estas señalan la importancia de la asistencia como predictora del desempeño académico. Aquí algunas de estas posturas:

Romer (1993), afirma que existe un "ausentismo exagerado" en los cursos de economía de pregrado en las principales universidades americanas. Además, existe una relación estadística muy estrecha entre el absentismo y el rendimiento, y la evidencia es consistente con la opinión de que esta relación tiene un componente causal importante.

Igual opinión presentan Yao y Chiang (2011), quienes por medio de un estudio de correlación de Pearson entre la asistencia a clase y las calificaciones globales en clases de informática encontraron una alta correspondencia entre las dos variables, concluyendo que estudiantes que no asistieron a algunas clases, especialmente las primeras, obtuvieron menores promedios de calificación que estudiantes que sí asistieron.

Bartual y Poblet (2009), explican que la continuidad en la asistencia incrementa el porcentaje de aprobación de un curso. Estudiantes que asisten a más del $80 \%$ de las clases se encuentran entre el $90 \%$ de los sujetos aprobados y notables, lo que determina que la asistencia tiene un grado alto de injerencia en la etapa de aprendizaje y su resultado académico. Conclusiones similares fueron señaladas por Park y Kerr (1990) y Crawford, Dale y Toney-McLin (2003), en donde se mostraba una fuerte asociación entre asistencia / ausentismo y el posterior éxito / fracaso académico en la asignatura. Incluso hasta en la propia relación asistencia-valoración del profesorado y el proceso académico (Expósito y De la Iglesia-Villasol, 2010).

De la misma forma, Pérez y Graell (2004), a través del coeficiente de correlación de Spearman, logran determinar una asociación positiva entre asistencia y rendimiento académico. Según ellos, dicha relación no solo afecta las asignaturas de los cursos básicos sino también a la de los cursos de profundización. Sin embargo, llaman la atención acerca del hecho de no poder extraer relaciones causales, por tanto, no se puede asegurar que ir a clase mejore el rendimiento, pues quizás la relación entre asistencia y rendimiento venga mediatizada por el tipo de estudiante.

Por su parte, Delgado-Hurtado y Castrillo-Lara (2015), identificaron que la dedicación del alumnado y la asistencia a clase tienen una incidencia positiva en los resultados alcanzados por el estudiantado, pues la dedicación y la asistencia son fundamentales para que se entere de las instrucciones relacionadas con los 
trabajos asignados en horarios por fuera de clase y con los posteriores exámenes, con lo que se puede establecer que dichos factores deben ser considerados como variables explicativas del éxito académico.

Igualmente, Landin y Pérez (2015) y Chen y Okediji (2014), en las áreas de medicina y economía, respectivamente, observaron relaciones positivas entre la asistencia y el rendimiento académico, a través de correlaciones estadísticamente significativas, donde estudiantes con una alta asistencia obtuvieron calificaciones más altas que quienes presentaron baja o ninguna asistencia.

Para finalizar, Borsato y Alves (2015), así como Trench (2001), afirman que no existen estudios en los cuales se sustente, de manera robusta, que la obligatoriedad en la asistencia redunde en un mayor rendimiento.

\section{LA PROPUESTA}

Como se ha evidenciado, la asistencia a clases como factor condicionante del desempeño académico individual ha llamado la atención de personal académico en diferentes universidades a nivel mundial. Generalmente, las políticas sobre asistencia se mueven entre los estadios de autonomía total o de obligatoriedad.

Es por eso que, a partir de la revisión anterior y con base en la necesidad de contextualizar el fenómeno en la realidad de la universidad, se propone realizar un estudio tendiente a abordar, entre otros, los siguientes aspectos:

- Influencia de la asistencia a clases en el rendimiento académico.

- Correspondencia entre niveles de asistencia y éxito o fracaso académico.

- Impacto de los factores de contexto en el rendimiento académico individual.

Para dicha indagación, se ha diseñado un estudio exploratorio y descriptivo, que intenta generar un espacio de reflexión académica sobre estos temas, los cuales impactan directamente la calidad del proceso de enseñanza-aprendizaje.

\section{Metodología}

\section{Sujetos}

Los sujetos son estudiantes de pregrado que cursaban asignaturas de primeros semestres (primero a cuarto) en una universidad privada colombiana (Universidad Autónoma de Occidente), en el periodo 2012, y de los cuales se tenían registros sobre sus niveles de asistencia en 213 asignaturas de carácter obligatorio. Esto corresponde a 7050 puestos estudiante (se utiliza el concepto de puesto estudiante, dado que un estudiante puede cursar más de una asignatura).

Es de resaltar que la población objeto de estudio se comporta de manera homogénea intra-grupos, por lo tanto, la dispersión es mínima por las áreas disciplinares analizadas. Esta situación permite la abstracción y generalización de conclusiones que representan las características de la problemática en el contexto total de la universidad seleccionada.

\section{Fuentes UTILIZADAS}

Acceso a bases de datos institucionales en las cuales reposa información asociada a asignaturas y estudiantes: niveles de mortalidad, satisfacción general con el desempeño docente en un curso, niveles de asistencia, nota definitiva obtenida, exámenes parciales, tamaño del curso, etc. 
El uso que se le dio a estas fuentes estuvo fundamentado en una aproximación de carácter descriptivo, que permitió, en primera instancia, desarrollar tablas de frecuencias y establecer una caracterización de los sujetos estudiados en función de las variables anteriormente señaladas.

Para ello, se utilizó la ayuda del paquete computacional SPSS para estructurar no solamente las tablas sino también una primera propuesta de modelo funcional, en el que el rendimiento académico fungía como variable dependiente y el resto de variables correspondían a la parte independiente de la propuesta metodológica.

\section{Desarrollo}

A partir del estudio se intentó identificar, nombrar, comparar, clasificar, reconocer los elementos y establecer relaciones en torno al posible impacto que tendrían los niveles de asistencia en el rendimiento académico de estudiantes (en este ejercicio se asume rendimiento académico como las asignaciones numéricas o notas obtenidas por estudiantes al final del curso [GPA - Grade Point Average]). Dado que los contextos son diferentes, como diferentes son los estudiantados, profesorados y, en general, la universidad, no bastó con reconocer el éxito o fracaso de los modelos de asistencia voluntaria u obligatoria e implantarlos institucionalmente. Se debió, por tanto, realizar un estudio experimental que verificara las condiciones de operación en la Institución.

Para tal fin se propuso:

- Seleccionar un periodo de análisis, en el cual se tuvieran los registros de asistencia para las asignaturas obligatorias, así como lo rendimientos de cada estudiante a través de sus respectivas notas definitivas

- En el periodo predeterminado, cuantificar el impacto de la medida de asistencia obligatoria a través del número de eventos de asignaciones de 1.5 y el número de estudiantes que tuvieran dicha nota, por programa y por departamento académico de origen de las asignaturas.

- Determinar la correlación existente entre los niveles de inasistencia y la nota definitiva. Presumiblemente esta relación se comportaría de manera inversamente proporcional. El análisis debió ser realizado de manera global, a nivel de departamento académico y por cada asignatura con la característica de asistencia obligatoria

- Con base en la revisión bibliográfica, se exploraron variables adicionales del contexto académico que pudieran causar algún impacto en las notas definitivas.

- Se desarrolló una estimación de los pesos (so verificación de supuestos básicos) de las variables introducidas a través de un modelo de regresión lineal múltiple. El análisis debió ser realizado de manera global, a nivel de departamento académico y por cada asignatura con la característica de asistencia obligatoria. Dicho modelo se expresó con base en la siguiente ecuación:

\section{ECUACIÓN}

$$
Y=\beta_{0}+\beta_{1} X_{1}+\beta_{2} X_{2}+\cdots+\beta_{\mathrm{k}} X_{\mathrm{k}}
$$

En la anterior expresión, la variable dependiente $\mathrm{Y}$ es interpretada como una combinación lineal de Xk variables independientes, acompañadas de su peso relativo a través del coeficiente $\beta \mathrm{k}$. 


\section{Resultados}

La política y puesta en marcha del esquema de asistencia obligatoria, indudablemente ha tenido un impacto cuantificable en los rendimientos estudiantiles. La normativa de la Universidad Autónoma de Occidente establece que para asignaturas predeterminadas es imperativo asistir al menos al $80 \%$ de las sesiones programadas. Cualquier incumplimiento injustificado a lo anterior acarrea automáticamente la asignación de 1.5 como nota definitiva.

Durante el periodo de análisis (2012) se presentaron 750 eventos de incumplimiento a la normatividad, motivo por el cual 430 estudiantes $(6.1 \%$ del total) obtuvieron una o más notas definitivas de 1.5 (1.79 eventos / estudiante).

Un 63\% de estudiantes tuvo una sola asignación por este motivo; mientras, llama la atención, que aproximadamente uno de cada cinco estudiantes tiene tres o más notas de 1.5 registradas. Por ejemplo, Cine y Comunicación Digital, Contaduría Pública y Administración de Empresas son los programas con mayor tasa de pérdida por motivo de inasistencia.

\section{EXPLORACIÓN PRELIMINAR}

En la primera fase, se plantea un análisis institucional de correlación bivariada, con el fin de determinar de manera preliminar si existe algún tipo de asociación entre los niveles de inasistencia y los rendimientos académicos obtenidos por estudiantes. Vale la pena resaltar que la hipótesis a validar es la existencia de una relación inversamente proporcional. En otras palabras, a menores niveles de inasistencia, el estudiantado es proclive a experimentar mejores rendimientos.

En esta medida, se ha hecho un primer análisis exploratorio (Tabla 1), consistente en determinar las correlaciones bivariadas (a través del estimador de Pearson) por las asignaturas pertenecientes a cada departamento académico y en estudiantes por programa académico, en función de las variables inasistencia y nota definitiva, notándose en todos los casos una relación inversamente proporcional (excepto en un departamento académico, aunque la significancia de esta relación no permite que esta condición sea concluyente). 
TABLA 1

Asociaciones entre inasistencia y nota definitiva - departamento académico

\begin{tabular}{lll}
\hline Departamento académico & Asociación & Sig. (Bilateral) \\
\hline Centro de Innovación Educativa en Ingeniería & -0.385 & 0.000 \\
\hline Automática y Electrónica & 0.001 & 0.993 \\
\hline Ciencias Administrativas & -0.263 & 0.000 \\
\hline Ciencias Ambientales & -0.270 & 0.000 \\
\hline Ciencias de la Comunicación & -0.311 & 0.000 \\
\hline Ciencias Económicas & -0.280 & 0.000 \\
\hline Ciencias Sociales & -0.105 & 0.009 \\
\hline Energética y Mecánica & -0.241 & 0.000 \\
\hline Física & -0.202 & 0.000 \\
\hline Humanidades & -0.210 & 0.000 \\
\hline Lenguaje & -0.302 & 0.000 \\
\hline Matemáticas & -0.214 & 0.000 \\
\hline Operaciones y Sistemas & -0.150 & 0.000 \\
\hline Publicidad y Diseño & -0.272 & 0.000 \\
\hline Idiomas & -0.336 & 0.000 \\
\hline
\end{tabular}

Las asociaciones que se expresan (Tabla 2), aunque inversas, son muy bajas para tener indicios de que los niveles de asistencia parecieran impactar los resultados. El nivel de agregación es tal, que cursos en los que las asociaciones sean significativas son "opacados" por aquellos en los cuales las relaciones sean bajas o nulas. Sin embargo, el análisis anterior es valioso en tanto permite conocer, intuitivamente, por primera vez, la naturaleza de la relación inversamente proporcional. 
TABLA 2

Asociaciones entre inasistencia y nota definitiva - programa académico

\begin{tabular}{lll}
\hline Programa académico & Asociación & Sig. (Bilateral) \\
\hline Administración de Empresas & -0.203 & 0.000 \\
\hline Administración Ambiental & -0.218 & 0.000 \\
\hline Banca y Finanzas & -0.271 & 0.000 \\
\hline Cine y Comunicación Digital & -0.333 & 0.000 \\
\hline Comunicación Publicitaria & -0.271 & 0.000 \\
\hline Comunicación Social - Periodismo & -0.328 & 0.000 \\
\hline Contaduría Pública & -0.138 & 0.000 \\
\hline Diseño de la Comunicación Gráfica & -0.341 & 0.000 \\
\hline Economía & -0.191 & 0.000 \\
\hline Ingeniería Multimedia & -0.197 & 0.000 \\
\hline Ingeniería Ambiental & -0.142 & 0.001 \\
\hline Ingeniería Biomédica & -0.302 & 0.437 \\
\hline Ingeniería Eléctrica & -0.122 & 0.025 \\
\hline Ingeniería Electrónica & -0.168 & 0.014 \\
\hline Ingeniería Industrial & -0.190 & 0.000 \\
\hline Ingeniería Informática & -0.184 & 0.000 \\
\hline Ingeniería Mecánica & -0.191 & 0.000 \\
\hline Ingeniería Mecatrónica & -0.198 & 0.000 \\
\hline Mercadeo y Negocios Internacionales & -0.240 & 0.000 \\
\hline & &
\end{tabular}

Para contrarrestar lo anterior, se ha hecho un análisis al interior de cada curso dictado, tratando de identificar tendencias o patrones, teniendo como principal referente las posibles asociaciones entre las dos variables. A continuación, algunos hallazgos importantes:

- De las 534 asignaturas dictadas en el periodo objeto de análisis, 213 tuvieron el carácter de asistencia obligatoria, en cuyo caso se cuenta con los registros de inasistencia individuales y las respectivas notas definitivas.

- De estas 213 asignaturas, coeficientes de correlación inferiores a -0.5 se presentan en 30 de ellas. Esto implica que relaciones leves se presenten entre los niveles de inasistencia y las posteriores notas definitivas.

Por facultades, pertenecientes a la Universidad Autónoma de Occidente, estas asignaturas son:

a) Facultad de Ciencias Económicas y Administrativas: Activos (-0.539 sig. 0.003); Contabilidad Financiera y Bancaria (-0.645 sig. 0.000); Contabilidad Administrativa (-0.500 sig. 0.000); Estructura del Sistema y Mercados Financieros (-0.758 sig. 0.000) y Teoría Organizacional 2 (-0.535 sig. 0.000).

b) Facultad de Comunicación Social: Comunicación Integrada (-0.740 sig. 0.000); Comunicación Publicitaria 1 (-0.607 sig. 0.000); Comunicación y Cine (-0.636 sig. 0.005); Dirección de Sonido (-0.530 sig. 0.004); Diseño Básico (Publicitaria) (-0.775 sig. 0.000); Diseño Básico 2 (-0.582 sig. 0.000); Fotografía (-0.554 sig. 0.002); Fotografía 1 (-0.530 sig. $0.000)$; Historia del Cine 3 (-0.588 sig. 0.005); Imagen y Sonido Digital (-0.636 sig. 0.008); 
Informática Gráfica (-0.548 sig. 0.000); Introducción a la Comunicación Publicitaria (-0.585 sig. 0.000); Introducción al Campo Cinematográfico (-0.602 sig. 0.001); Introducción al Diseño Gráfico (-0.676 sig. 0.000); Narración y Argumentación (-0.583 sig. 0.004); Producción de Impresos (-0.583 sig. 0.000) y Teoría Cinematográfica (-0.527 sig. 0.002).

c) Facultad de Ciencias Básicas: Cartografía y Sensores Remotos (-0.504 sig. 0.033) y Ordenamiento Territorial (-0.529 sig. 0.002).

d) Facultad de Ingeniería: Estructura y Propiedades de los Materiales (-0.626 sig. 0.001); Gestión de Producto (-0.564 sig. 0.000) y Procesos y Materiales (-0.603 sig. 0.000).

e) Facultad de Humanidades: Ciencia y Religión (-0.538 sig. 0.000); Ecosofía y Diversidad Cultural (-0.509 sig. 0.000) y Música e Identidad Cultural (-0.511 sig. 0.000).

Como se puede observar, la mayoría de coeficientes se encuentran en el intervalo de - 0.5 a - 0.7 ; situación que deja entrever asociaciones moderadas leves. En el presente análisis, como se mostró anteriormente, pocas asignaturas cumplen con esta propiedad; en el resto simplemente la asociación es no significativa o nula. Esto implica que los niveles de inasistencia en las asignaturas analizadas son, en efecto, un condicionante alrededor de las posteriores notas definitivas, aunque, como se puede evidenciar, en la contextualización inicial de este documento, la mayoría de las asignaturas se encuentra en el intervalo correspondiente a primero a cuarto semestre, lo que culturalmente implica que el estudiantado está pasando por su periodo de transición entre la educación media y la superior.

En este sentido, se empiezan a fomentar, desde el punto de vista pedagógico, enfoques en los que el estudiantado es autónomo en su proceso formativo y la asistencia a clases pasa de imponerse como obligatoria a ser un acto de potestad del individuo dentro de su propio proceso de aprendizaje.

\section{VARIABLES DE CONTEXTO ACADÉMiCO}

Estudiantado económicamente desfavorecido, por ejemplo, en Canadá, es menos susceptible de continuar sus estudios universitarios si se comparan con estudiantes provenientes de familias adineradas. Cerca del 96\% de diferencia en los niveles de asistencia total a la universidad son explicados a partir del cuartil de ingreso de los padres (Frenette, 2007). Existe, por tanto, una prevalencia de factores socioeconómicos del estudiantado, como variable independiente, en posibles registros de asistencia a clase, así como existen factores del contexto académico que pueden influir tanto en los niveles de asistencia como en los rendimientos académicos observados, de manera simultánea.

Con base en lo anterior, se han adicionado una serie de variables que ayudan a fortalecer la estimación, tratando de minimizar la no inclusión de elementos ocultos y que podrían llegar a tener alguna incidencia. En este sentido, se ha involucrado en el estudio la satisfacción general que estudiantes tengan con el trabajo desarrollado por el personal docente, el número de estudiantes del curso y la mortalidad histórica del curso; variables de contexto académico que pueden impactar en los procesos de motivación del estudiantado; todo lo cual podría provocar el incremento en los niveles de inasistencia. Al respecto, Pintrich (1994), afirma que el contexto de clase, los sentimientos de estudiantes, sus creencias y sus comportamientos son los elementos motivacionales inherentes a una clase.

Se identificaron a nivel inicial, dos relaciones directamente proporcionales y dos inversamente proporcionales (Tabla 3). Es de anotar que dichas relaciones son casi nulas desde el punto de vista lineal. De esta manera, se esperaría que estudiantes con mayores niveles de inasistencia y asignaturas con tasas de mortalidad históricas más altas reviertan en notas definitivas individuales menores. Mayor tamaño de curso y mayor satisfacción con el desempeño docente presumiblemente traería como consecuencia mejores rendimientos individuales. En este caso, solo se ha podido conocer la dirección de la relación, aunque como se puede evidenciar, las asociaciones son prácticamente nulas. 
TABLA 3

Asociaciones teniendo en cuenta variables adicionales

\begin{tabular}{|c|c|c|c|c|c|c|}
\hline & & $\begin{array}{l}\text { Nota } \\
\text { definitiva }\end{array}$ & Inasistencia & $\begin{array}{l}\text { Satisfacción } \\
\text { general }\end{array}$ & $\begin{array}{l}\text { Número de } \\
\text { estudiantes }\end{array}$ & Mortalidad \\
\hline \multirow[t]{4}{*}{$\begin{array}{l}\text { Nota } \\
\text { definitiva }\end{array}$} & $\begin{array}{l}\text { Correlación de } \\
\text { Pearson }\end{array}$ & 1,000 & $-205^{* *}$ & $.113^{* *}$ &, $144^{\text {** }}$ &,$- 378^{* *}$ \\
\hline & Sig. (bilateral) & , &, 000 &, 000 &, 000 &, 000 \\
\hline & $\mathrm{N}$ & & & & & \\
\hline & & 20182 & 20182 & 16996 & 16996 & 19939 \\
\hline \multirow[t]{4}{*}{ Inasistencia } & $\begin{array}{l}\text { Correlación de } \\
\text { Pearson }\end{array}$ &,$- 205^{* *}$ & 1,000 &, 009 &, $092^{* *}$ &,$- 106^{* *}$ \\
\hline & Sig. (bilateral) &, 000 & . &, 221 & .000 & .000 \\
\hline & $\mathrm{N}$ & & & & & \\
\hline & & 20182 & 22022 & 18576 & 18576 & 21769 \\
\hline \multirow[t]{4}{*}{$\begin{array}{l}\text { Satisfacción } \\
\text { general }\end{array}$} & $\begin{array}{l}\text { Correlación de } \\
\text { Pearson }\end{array}$ & $.113^{* *}$ & .009 & 1,000 &, $075^{* \approx}$ &,- 038 \\
\hline & Sig. (bilateral) & .000 &, 221 & , &, 000 &, 000 \\
\hline & $\mathrm{N}$ & & & & & \\
\hline & & 16996 & 18576 & 18576 & 18576 & 18389 \\
\hline \multirow[t]{4}{*}{$\begin{array}{l}\text { Número de } \\
\text { estudiantes }\end{array}$} & $\begin{array}{l}\text { Correlación de } \\
\text { Pearson }\end{array}$ &, $144^{* \star}$ &, $092^{* *}$ &, $075^{* *}$ & 1,000 & $\cdot 346^{* *}$ \\
\hline & Sig. (bilateral) &, 000 &, 000 &, 000 & , &, 000 \\
\hline & $\mathrm{N}$ & & & & & \\
\hline & & 16996 & 18576 & 18576 & 18576 & 18389 \\
\hline \multirow[t]{4}{*}{ Mortalidad } & $\begin{array}{l}\text { Correlación de } \\
\text { Pearson }\end{array}$ &, $378^{* *}$ &,$- 106^{\star *}$ &,$- 038^{* *}$ & $\cdot 346^{* *}$ & 1,000 \\
\hline & Sig. (bilateral) &, 000 &, 000 &, 000 &, 000 & , \\
\hline & $\mathrm{N}$ & & & & & \\
\hline & & 19939 & 21769 & 18389 & 18389 & 21769 \\
\hline
\end{tabular}

Nota: *" Asociación altamente significativa.

** Asociación altamente significativa

\section{EL MODELO}

Intuitivamente se ha notado, a través del coeficiente de correlación de Pearson, que no existe una asociación tal, que alguna de las cinco variables consideradas sea explicada por otra de ellas. Estas variables interactúan en un contexto de clase y pudieran tener un impacto directo en las notas definitivas.

Desde el punto de vista educativo, esta no explicación de las variables por las otras está generando la necesidad de comprender el entorno de aprendizaje como un espacio de convergencia de aspectos complementarios; pero irreductibles, dado el carácter complejo que los procesos educativos conllevan. Por esto, es imposible pensar en la asistencia a clases como un factor aislado de aspectos como: el tamaño del curso o la valoración que hace el estudiantado del trabajo docente. Como se puede evidenciar, todos estos elementos pueden condicionar el desempeño académico, pero son unidades complejas cuya valoración debe hacerse en el marco de la integralidad sin perder la especificidad.

$\mathrm{Al}$ respecto, se ha caracterizado esta situación a través de un modelo de regresión lineal múltiple, encontrando los siguientes resultados concretos:

Tomadas juntas, las cuatro variables independientes (inasistencia a clase, satisfacción general con el desempeño del profesorado, número de estudiantes matriculados y matriculadas en el curso, mortalidad) en el análisis explican un 22.3\% de la varianza de la variable dependiente (nota definitiva). Esto indica que, sin duda, las variables incluidas son importantes, pero que aún falta conocer aproximadamente un $80 \%$ a través de otros factores que todavía no están siendo tenidos en cuenta, probablemente: rendimientos académicos previos, motivación natural hacia al estudio o condiciones socioeconómicas del estudiantado. De la misma forma, se puede asumir independencia entre los residuos, dado que el estadístico Durbin-Watson toma valores entre 1.5 y 2.5 (supuesto de independencia) (Tabla 4). 
TABLA 4

Modelo de regresión múltiple para la Institución

\begin{tabular}{cccccc}
\hline Modelo & R & R cuadrado & $\begin{array}{c}\text { R cuadrado } \\
\text { corregida }\end{array}$ & $\begin{array}{c}\text { Error tip. de } \\
\text { la estimacion }\end{array}$ & $\begin{array}{c}\text { Durbin- } \\
\text { Watson }\end{array}$ \\
\hline 1 &, 472 &, 223 &, 223 &, 5844 & 1,585 \\
\hline
\end{tabular}

El estadístico F (Tabla 5) contrasta la hipótesis nula de que el valor poblacional de $\mathrm{R}$ es cero y, por tanto, permite vislumbrar la existencia de relación lineal significativa entre la variable dependiente y el conjunto de variables independientes tomadas juntas. El valor del nivel crítico sig. $=0.000$ indica que sí existe relación lineal significativa. Por tanto, el hiperplano definido por la ecuación de regresión ofrece un buen ajuste a la nube de puntos.

TABLA 5

Resumen del ANOVA

\begin{tabular}{cccccc}
\hline Modelo & $\begin{array}{c}\text { Suma de } \\
\text { cuadrados }\end{array}$ & gl & $\begin{array}{c}\text { Media } \\
\text { cuadrática }\end{array}$ & F & Sig. \\
\hline 1 & Regresión & 1649,989 & 4 & 412,497 &, 000 \\
& Residual & 5741,782 & 16814 &, 341 & \\
& Total & 7391,770 & 16818 & & \\
\hline
\end{tabular}

La ecuación de regresión mínimo cuadrática toma la siguiente forma:

\section{ECUACIÓN DE REGRESIÓN}

Nota definitiva $=3.574-3.15 \mathrm{E} \cdot 02 \times$ Inasistencia $+5.446 \mathrm{E} \cdot 03 \times$ Satisfacción general $+5.595 \mathrm{E}-03 \times$ Número de estudiantes $-1.948 \times$ Tasa de mortalidad

La ecuación de regresión indica que la mayor influencia en las posteriores notas definitivas (acorde con el porcentaje de varianza explicada), es debido en gran medida a la tasa de mortalidad histórica de la asignatura. Estos coeficientes son dependientes unos de otros, por lo que se denominan, a menudo, coeficientes de regresión parcial, debido a que el valor estimado para cada uno se determina con base en el análisis del resto de variables independientes.

En función de los coeficientes estandarizados, estos están basados en las puntuaciones típicas, situación que los hace comparables, lo que permite inferir el cambio en las puntuaciones típicas de la variable dependiente, como resultado del cambio unitario en la variable independiente, se mantiene constante el resto de variables. En este caso, una influencia menor es percibida (como ya se mencionó anteriormente) por los registros históricos de mortalidad de la asignatura y los niveles de inasistencia individual, ambos aspectos en orden inversamente proporcional (Tabla 6).

TABLA 6

Coeficientes de correlación parcial

\begin{tabular}{|c|c|c|c|c|c|c|}
\hline \multirow[b]{2}{*}{ Modelo } & & \multicolumn{2}{|c|}{$\begin{array}{l}\text { Coeficientes no } \\
\text { estandarizados }\end{array}$} & \multirow{2}{*}{$\begin{array}{c}\begin{array}{c}\text { Coeficientes } \\
\text { estandarizados }\end{array} \\
\text { Beta }\end{array}$} & \multirow[b]{2}{*}{$\mathbf{t}$} & \multirow[b]{2}{*}{ Sig. } \\
\hline & & B & Error típ. & & & \\
\hline \multirow[t]{5}{*}{1} & (Constante) & 3,574 &, 039 & & 91,364 &, 000 \\
\hline & Inasistencia & $-3,15 \mathrm{E} \cdot 02$ &, 001 & $\cdot, 268$ & $-39,003$ & 000 \\
\hline & Satisfacción general & $5,446 \mathrm{E} \cdot 03$ & .000 &, 103 & 15,075 &, 000 \\
\hline & Número de estudiantes & $5,595 \mathrm{E} \cdot 03$ & .001 & .048 & 6,642 &, 000 \\
\hline & Mortalidad & $-1,948$ & .036 & $\cdot, 392$ & $-54,685$ &, 000 \\
\hline
\end{tabular}


Para cada uno de los departamentos académicos se corrió un modelo de regresión lineal múltiple de características similares (Tabla 7):

TABLA 7

Modelos de regresión múltiple por departamento académico

\begin{tabular}{|c|c|c|c|}
\hline $\begin{array}{l}\text { Departamento } \\
\text { académico }\end{array}$ & R2 (corregida) & Sig. F & Expresión \\
\hline $\begin{array}{l}\text { Centro de Innovación } \\
\text { Educativa en Ingeniería }\end{array}$ & 0.184 & 0.000 & $\begin{array}{l}2.407-4.64 \mathrm{E} \cdot 02 \times \text { Inasistencia }+1.357 \mathrm{E} \cdot 03 \times \\
\text { Satisf }+5.465 \mathrm{E}-02 \times \text { \# Est. }-5.94 \mathrm{E} \cdot 02 \times \text { Mortalidad }\end{array}$ \\
\hline Automática y Electrónica & 0.146 & 0.000 & $\begin{array}{l}3.671-4.06 \text { E- } 02 \times \text { Inasistencia }-5.65 E-04 \times \text { Satisf. } \\
+2.759 \text { E- } 03 \times \text { \# Est }-0.687 \times \text { Mortalidad }\end{array}$ \\
\hline Ciencias Administrativas & 0.075 & 0.000 & $\begin{array}{l}3.989-2.39 \mathrm{E} \cdot 02 \times \text { Inasistencia + } 1.491 \mathrm{E} \cdot 03 \times \\
\text { Satisf. - 3.24E-03 } \times \text { \# Est. }-1.344 \times \text { Mortalidad }\end{array}$ \\
\hline Ciencias Ambientales & 0.260 & 0.000 & $\begin{array}{l}3.590-3.22 \mathrm{E}-02 \times \text { Inasistencia }+6.884 \mathrm{E} \cdot 03 \mathrm{x} \\
\text { Satisf. }-1.21 \mathrm{E} \cdot 03 \mathrm{x} \text { \# Est. }-2.761 \mathrm{x} \text { Mortalidad }\end{array}$ \\
\hline Ciencias de la Comunicación & 0.172 & 0.000 & $\begin{array}{l}\text { 4.304-3.04E-02 X Inasistencia +6.108E-03 } \mathrm{x} \\
\text { Satisf. - 9.49E-03 X \# Est. }-3.602 \times \text { Mortalidad }\end{array}$ \\
\hline Ciencias Económicas & 0.139 & 0.000 & $\begin{array}{l}3.273-4.40 \mathrm{E}-02 \times \text { Inasistencia }+3.879 \mathrm{E} \cdot 03 \mathrm{x} \\
\text { Satisf }+1.593 \mathrm{E} \cdot 02 \mathrm{x} \text { \# Est. }-1.211 \times \text { Mortalidad }\end{array}$ \\
\hline Ciencias Sociales & 0.147 & 0.000 & $\begin{array}{l}2.945-2.08 \mathrm{E}-02 \times \text { Inasistencia }+9.703 \mathrm{E}-03 \times \text { Satisf. } \\
+1.584 \mathrm{E}-02 \times \# \text { Est. }-2.259 \times \text { Mortalidad }\end{array}$ \\
\hline Energética y Mecánica & 0.252 & 0.000 & $\begin{array}{l}2.992-2.11 \mathrm{E} \cdot 02 \times \text { Inasistencia }+1.083 \mathrm{E}-02 \times \text { Satisf. } \\
+2.256 \mathrm{E} \cdot 02 \mathrm{x} \text { \# Est. }-2.217 \times \text { Mortalidad }\end{array}$ \\
\hline Fisica & 0.112 & 0.000 & $\begin{array}{l}2.473-3.25 \mathrm{E}-02 \times \text { Inasistencia }+8.911 \mathrm{E}-03 \times \text { Satisf. } \\
+1.287 \mathrm{E}-02 \times \# \text { Est. }-0.622 \times \text { Mortalidad }\end{array}$ \\
\hline Humanidades & 0.112 & 0.000 & $\begin{array}{l}3.253-2.84 \mathrm{E} \cdot 02 \times \text { Inasistencia }+2.846 \mathrm{E} \cdot 03 \times \\
\text { Satisf. }+1.647 \mathrm{E} \cdot 02 \times \text { \# Est. }+1.530 \times \text { Mortalidad }\end{array}$ \\
\hline Lenguaje & 0.145 & 0.000 & $\begin{array}{l}3.681-3.53 \mathrm{E}-02 \times \text { Inasistencia }+8.852 \mathrm{E}-03 \mathrm{x} \\
\text { Satisf. - } 1.26 \mathrm{E}-02 \mathrm{x} \text { \# Est. - } 1.057 \times \text { Mortalidad }\end{array}$ \\
\hline Matemáticas & 0.077 & 0.000 & $\begin{array}{l}3.575-2.80 \mathrm{E}-02 \times \text { Inasistencia }+5.114 \mathrm{E}-03 \times \text { Satisf. } \\
-5.17 \mathrm{E}-03 \mathrm{x} \# \text { Est. }-1.350 \times \text { Mortalidad }\end{array}$ \\
\hline Operaciones y Sistemas & 0.108 & 0.000 & $\begin{array}{l}\text { 4.280-3.16E- } 02 \times \text { Inasistencia }+3.891 \mathrm{E}-03 \times \text { Satisf. } \\
-2.30 \mathrm{E}-02 \times \text { \# Est. }-2.113 \times \text { Mortalidad }\end{array}$ \\
\hline Publicidad y Diseño & 0.181 & 0.000 & $\begin{array}{l}3.860-3.69 \mathrm{E}-02 \times \text { Inasistencia }+3.530 \mathrm{E}-03 \mathrm{x} \\
\text { Satisf. }+9.475 \mathrm{E}-03 \mathrm{x} \text { \# Est. }-2.830 \times \text { Mortalidad }\end{array}$ \\
\hline Idiomas & 0.134 & 0.000 & $\begin{array}{l}2.685-2.77 \mathrm{E} \cdot 02 \times \text { Inasistencia }+6.328 \mathrm{E} \cdot 03 \times \\
\text { Satisf }+1.693 \mathrm{E} \cdot 02 \times \text { \# Est. }+0.928 \times \text { Mortalidad }\end{array}$ \\
\hline
\end{tabular}

En cada uno de estos casos, los modelos fueron validados en términos de los supuestos básicos, y se encontró que el porcentaje de varianza explicada de las notas definitivas en función de las cuatro variables era pequeño, volviendo a poner en discusión la necesidad de explorar sobre más aspectos que pueden condicionar el rendimiento de una asignatura.

En todos los casos, los modelos presentados rechazan la hipótesis nula de $\mathrm{R}=0$; es decir, sí existe algún patrón lineal entre la variable dependiente y las cuatro empleadas como independientes.

Llama la atención que cuando se determinan los coeficientes o pesos de las variables en el modelo, y tomando en consideración el sentido de la magnitud, las variables de mortalidad histórica de la asignatura e inasistencia presentan relaciones inversamente proporcionales; es decir, niveles de mortalidad histórica de la asignatura mayores e inasistencias individuales superiores tendrían alguna disminución en la nota definitiva. Esta afirmación solamente deja entrever el sentido, pero como ya se indicó no existen evidencias contundentes para determinar que sí existe siquiera una asociación moderada entre las variables (coeficiente de determinación R2).

Esto implicaría que el nivel de dificultad de la asignatura incrementa progresivamente la posibilidad de fracaso si se incorpora el hecho de las faltas, lo que ocasionaría como lineamiento de futuras políticas condicionar los porcentajes de asistencia con un mayor énfasis hacia las asignaturas que históricamente han representados niveles de complejidad superiores para el estudiantado.

Cuando se hace el análisis desagregado para cada asignatura dictada en el periodo, se observa que el impacto de las variables mencionadas en una posible nota definitiva es bajo o en algunos casos nulo. Esto implicaría que las variables del contexto académico, entre ellas los niveles de inasistencia, no serían tan determinantes a la hora de medir la efectividad del proceso de enseñanza-aprendizaje. Otra conclusión derivada apunta 
a considerar variables endógenas al estudiantado, como los procesos de motivación natural o asistida al aprendizaje, condiciones socieconómicas propias o de las familias, entorno laboral, etc.

\section{Discusión}

A la luz de los resultados obtenidos se plantean algunos elementos de reflexión en torno al alcance de la investigación, así como de futuros desarrollos:

\section{DeL ESTUDio}

La instauración de la política se ha realizado para asignaturas predeterminadas, sin estudios previos de pilotaje para determinar posibles impactos. En este sentido, la exploración de las variaciones en las notas definitivas se encuentra supeditada a aquel estudiantado con niveles de asistencia iguales o superiores a $80 \%$, y deja para estudiantes con asistencias menores automáticamente la nota definitiva de 1.5 (para eliminar el sesgo estos grupos de estudiantes fueron eliminados de los modelos corridos, dado que el reflejo siempre iba a ser constante). Esto causa que el espectro se reduzca y las variaciones sean condicionadas a unos porcentajes específicos conocidos a priori.

Sería recomendable, para un periodo posterior, realizar estudios por muestreo estadístico para asignaturas en las cuales se tengan grupos de experimentación y control en cada departamento académico, y a través de esto determinar en el contexto de la Universidad el impacto de los niveles de asistencia en las notas definitivas, para poder controlar todos los posibles porcentajes de asistencia y su efecto en la escala de valoración final.

\section{DE LA DISPONIBILIDAD DEL PROFESORADO EN ESPACIOS FUERA DE CLASE}

Estudiantes que no asisten a clase tendrían oportunidad de consultar a sus docentes fuera del salón de clase sobre las temáticas abordadas y esto podría generar un ciclo en el cual en próximas ocasiones reincidan en la conducta. Postura contraria se presenta cuando esta conducta genera en el estudiantado un nivel de empatía con el profesorado, que termine por motivar el proceso de enseñanza-aprendizaje y sus niveles de asistencia a clase. Vale la pena, en próximas investigaciones, abordar la posibilidad o acceso a tutorías extra clase, por parte de estudiantes.

\section{DE LA MOTIVACIÓN NATURAL Y DE LA INDUCIDA}

La motivación natural se refiere a aquella conducta o conjunto de hábitos con los que viene el estudiantado antes de entrar a un curso universitario. Su disposición natural al aprendizaje y su historia académica son, sin duda, factores clave a la hora de determinar tanto el rendimiento como la asistencia a las sesiones de trabajo. Queda en las aulas la responsabilidad social por la segunda, y generar en sus estudiantes esa serie de "chispas al cerebro" que hace que el encuentro con el profesorado sea necesario, interesante, oportuno y efectivo. Es posible que la asistencia no sea un problema solo del estudiantado, tal vez los maestros y maestras debemos garantizar ambientes de aprendizaje que hagan de cada encuentro académico una experiencia única e irrepetible. 


\section{Conclusiones}

Con base en el estudio anterior se conocieron posturas antagónicas, divergentes y complementarias sobre la problemática de la asistencia a clases y su impacto en los rendimientos de estudiantes (GPA). Se revisó la bibliografía y se encontró una comunidad científica que respalda la necesidad imperiosa de realizar este tipo de estudios.

De acuerdo con lo presentado por autores como San Martín-Gutiérrez, Jiménez-Torres y JerónimoSánchez-Beato (2016), la medida de asistencia obligatoria carece de sentido siempre y cuando no exista un marco de directrices que fomente dicha asistencia más allá de un prerrequisito. En este sentido, los autores del presente artículo afirman que las medidas coercitivas poco o nada benefician al proceso de enseñanzaaprendizaje, más bien privilegian aspectos administrativos que terminan por deteriorar la motivación intrínseca y natural asociada al aprendizaje significativo, acorde con los planteamientos de Ausubel.

En el contexto de la universidad analizada, específicamente, se puede notar que las asignaciones de 1.5 por inasistencia, así como el porcentaje de estudiantes que merecieron esta aplicación, son importantes y requieren la atención de las directivas académicas. Esto, debido a que la fijación de los rangos de corte para penalizar la ausencia depende (en teoría) de cada conjunto de asignaturas, de sus campos disciplinares y del balance teórico-práctico que ostenten. Dicho de otro modo, son incipientes los estudios en el concierto global que atribuyen al nivel de complejidad de la asignatura los porcentajes de asistencia requeridos para alcanzar el éxito académico.

De la misma forma, se pudo abstraer que los niveles de asistencia en las personas estudiadas no presentaron asociación significativa con los desempeños académicos estudiantiles. Intuitivamente, se nota una relación inversamente proporcional entre los niveles de inasistencia y los posteriores rendimientos, aunque sin encontrar evidencia de asociaciones significativas moderadas o fuertes.

Desde lo planteado en el estado del arte de este estudio, se genera la hipótesis que define que, a menores niveles de inasistencia, mayor rendimiento académico; sin embargo, el estudio puede probar la relación inversa, pero no la magnitud de la asociación; es decir, se reconoce la importancia de la asistencia mas no su fuerza explicativa del rendimiento, situación que puede ser explicada por la política de asistencia obligatoria generada en la institución objeto de análisis.

$\mathrm{Al}$ respecto, al poner en consideración variables adicionales como: inasistencia a clase, satisfacción general con el desempeño del profesorado, número de estudiantes en el curso y mortalidad histórica de la asignatura, se logra explicar una pequeña variación de la nota definitiva; lo cual determina, de esta manera, que es menester abordar otra serie de variables exógenas al contexto puramente académico.

Para finalizar, el constructivismo educativo propone un paradigma donde el proceso de enseñanzaaprendizaje se asume como un ejercicio dinámico, participativo e interactivo de la relación sujeto dispuesto a aprender-sujeto que construye conocimiento en conjunto. Es desde esta perspectiva, que el condicionamiento propio de la teoría conductivista puede, a partir del estímulo de la obligatoriedad, generar una reacción que bien podría traer resultados en el rendimiento. No obstante, la obligatoriedad puede tratarse en tanto que variable espuria y, eventualmente, constituir el real estímulo la motivación natural del estudiantado hacia aprender y, por tanto, tener consecuencias en su asistencia como en su rendimiento, lo que plantearía la relevancia del enfoque constructivista en el aprendizaje de estudiantes, y generaría la necesidad insaciable del conocimiento.

\section{REFERENCIAS}

Bartual, T. y Poblet, M. C. (2009). Determinantes del rendimiento académico en estudiantes universitarios de primer año de economía. Revista de Formación e Innovación Educativa Universitaria, 2(3), 172-181. 
Berenson, S. B., Carter, G. y Norwood, K. S. (1992). The at-risk student in college developmental algebra [El estudiante universitario en situación de riesgo en el desarrollo del álgebra]. School Science and Mathematics, 92, 55-58.

Borsato, F. P. y Alves, J. M. (2015). Student assistance in higher education in Brazil [Asistencia de los estudiantes en la educación superior en Brasil]. Procedia - Social and Behavioral Sciences, 174, 1542-1549.

Chen, Q.y Okediji, T. O. (2014). What is behind class attendance in college economics courses? [¿Qué hay detrás de la asistencia a clases en los cursos de economía en la universidad?]. Applied Economics Letters, 21(6), 433-437.

Crawford, J., Dale, L. y Toney-McLin, P. (2003). Student performance factors in economics and accounting [Factores de rendimiento de los estudiantes de economía y contabilidad]. Allied Academies International Conference, Las Vegas, Estados Unidos.

Dayo, S. (2011). La gestion de l'absenteisme des eleves dans les lycees et colleges: strategies de traitement, consequences et perspectives [La gestión del absentismo de los estudiantes en las escuelas secundarias y universidades: Estrategias de tratamiento, consecuencias y perspectivas]. Koudougou: Université de Koudougou - École Normale Superieure.

Delgado-Hurtado, M. M. y Castrillo-Lara, L. A. (2015). Efectividad del aprendizaje cooperativo en contabilidad: una contrastación empírica. Revista de Contabilidad, 18(2), 138-147.

Expósito, E. G. y De la Iglesia-Villasol, M. C. (2010). Valoración de los profesores y asistencia a clase de los alumnos. ¿Existe relación causal? Investigaciones de Economía de la Educación, 50(5), 995-1016.

Frenette, M. (2007). Pourquoi les jeunes provenant de familles à plus faible revenue sont-ils moins susceptibles de fréquenter l'université? Analyse fondée sur les aptitudes aux études, l'influence des parents et les contraintes financières [¿ंPor qué los jóvenes de familias con menor respaldo tienen menos probabilidades de asistir a la universidad? Análisis de las habilidades académicas, la influencia de los padres y las limitaciones financieras]. Montreal: Statistique Canada - Government of Canada.

Hyde, R. M. y Flournoy, D. J. (1986). A case against mandatory lecture attendance [Un caso en contra de la asistencia obligatoria]. Journal of Medical Education, 61, 175-176.

Landin, M. y Pérez, J. (2015). Class attendance and academic achievement of pharmacy students in a European University [La asistencia a clase y el rendimiento académico de los estudiantes de farmacología en la universidad europea]. Currents in Pharmacy Teaching and Learning, 7(1), 78-83.

Levine, R. y Renelt, D. (1992). A sensitive analysis of cross country growth regressions [Un análisis sensible de las regresiones de crecimiento entre países]. The American Economic Review, 82 (4), 942-963.

Park, K. y Kerr, P. (1990). Determinants of academic performance: a multinomial logit approach [Determinantes del rendimiento académico: Un modelo logístico multinomial]. Journal of Economic Education, 21(2), 101-111.

Pérez, J. y Graell, S. (2004). Asistencia a clase y rendimiento académico en estudiantes de medicina. La experiencia de la Universidad Autónoma de Barcelona. Revista de Educación Médica, 7(2), 85-89.

Pintrich, P. R. (1994). Student motivation in the college classroom [La motivación del estudiante universitario en el salón de clases]. En K. W. Pritchard and R. McLaran Sawyer (Eds.), Handbook of college teaching: theory and application (pp. 23-24). Westport, CN: Greenwood Press.

Ramsden, P. (1991). A performance indicator of teaching quality in higher education [Un indicador de la calidad de la enseñanza en la educación superior]. Studies in Higher Education, 16(2), 129-140.

Romer, D. (1993). Do students go to class? [¿Los estudiantes asisten a clase?]. The Journal of Economic Perspectives, 7(3), 167-174.

San Martín-Gutiérrez, S., Jiménez-Torres, N. y Jerónimo-Sánchez-Beato, E. (2016). The evaluation of the university students in the European Higher Education Area [La evaluación de los estudiantes universitarios en el Espacio Europeo de Educación Superior]. Aula Abierta, 44(1), 7-14.

St. Clair, K. L. (1999). A case against compulsory class attendance policies in higher education [Un caso en contra las políticas de asistencia obligatoria a clases en la educación superior]. Innovative Higher Education, 23 (8), 171-180.

Trench, J. M. (2001). Asistencia y rendimiento académico. Revista Digital de Educación y Nuevas Tecnologías, 3(19).119-133. 
Jesús Gabalán Coello, et al. Rendimiento académico universitario y asistencia a clases: Una Visión...

Trigwell, K. y Prosser, M. (2000). Understanding learning and teaching: The experience in higher education [Comprendiendo el aprendizaje y la enseñanza: La experiencia en la educación superior]. Londres: The Society for Research into Higher Education.

Yao, J. F.J. y Chiang, T. M. (2011). Correlation between class attendance and grade [Correlación entre la asistencia a clases y las calificaciones]. Journal of Computing Sciences in Colleges, 27(2), 142-147.

\section{Notas}

[3] Este artículo expone resultados de una investigación denominada "Asistencia a clases como condicionante del desempeño académico", realizada en la Universidad Autónoma de Occidente, Colombia. Este derivó en un posterior análisis comparativo con la Universidad Tarapacá, Chile, cuyos resultados en el contexto chileno se presentaron en las Jornadas de Investigación e Innovación UTA 2014. Universidad de Tarapacá, Arica 7 y 8 de agosto de 2014.

\section{BY-NC-ND}

